This is an accepted manuscript to the 2019 IEEE International Conference on Prognostics and Health Management

The final manuscript can be found under the following link:

https://ieeexplore.ieee.org/abstract/document/8819445

(C) 2019 IEEE. Personal use of this material is permitted. Permission from IEEE must be obtained for all other uses, in any current or future media, including reprinting/republishing this material for advertising or promotional purposes, creating new collective works, for resale or redistribution to servers or lists, or reuse of any copyrighted component of this work in other works 


\title{
Estimating remaining useful life of machine tool ball screws via probabilistic classification
}

\author{
Maximilian Benker*, Robin Kleinwort ${ }^{\dagger}$, Michael F. Zäh ${ }^{\ddagger}$ \\ Institute for Machine Tools and \\ Industrial Management \\ Technical University Munich \\ Boltzmannstr. 15, 85748 Garching b. München \\ Email: *maximilian.benker@iwb.mw.tum.de, ${ }^{\dagger}$ robin.kleinwort@iwb.mw.tum.de, ${ }^{\dagger}$ michael.zaeh@iwb.mw.tum.de
}

\begin{abstract}
Ball screws are key components in machine tool linear feed drives since they translate the motors' rotary motion into linear motion. With usage over time, however, tribological degradation of ball screws and the successive loss in preload can cause imprecise position accuracy and loss in manufacturing precision. Therefore condition monitoring (CM) of ball screws is important since it enables just in time replacement as well as the prevention of production stoppages and wasted material. This paper proposes an idea based on a probabilistic classification approach to monitor a ball screw's preload condition with the help of modal parameters identified from vibration signals. It will be shown that by applying probabilistic classification models, uncertainties with respect to degradation can be quantified in an intuitive way and therefore can enhance the basis of decision making. Furthermore, it will be shown how a probabilistic classification approach allows the estimation of remaining useful life (RUL) for ball screws when the user only has access to discrete preload observations.
\end{abstract}

Keywords-Remaining useful life, machine tool, ball screw, probabilistic classification, Gaussian process.

\section{INTRODUCTION}

Machine tool operating costs can be divided into productivity costs such as labour and material, flexibility costs such as setup and waiting times and quality costs such as prevention of failure and follow-up costs related to failures [1]. Quality costs can make up a major part of the total costs since failures usually occur unexpectedly and therefore imply further correction costs. In some safety-critical cases, failures are not even tolerable. Due to that, many companies follow a preventative strategy, which implies maintaining on a regular schedule. This often means replacing parts regardless of their degradation condition, which ultimately leads to high replacement and maintenance costs [2]. Especially for machine tools with their vast life cycles, maintenance is a crucial aspect [3]. Therefore, a CM system that can estimate RUL accurately supports the user in making better maintenance decisions to prevent both unnecessary preventive and failure costs, and ultimately leads to an economic advantage in operating the machine tool.

The machine tool's feed drives, which determine the relative positioning of cutting tool and work piece, which in return has a huge impact on manufacturing accuracy, are maintenance intensive components and considered in this paper. Those feed drives are commonly realized by either linear or rotary

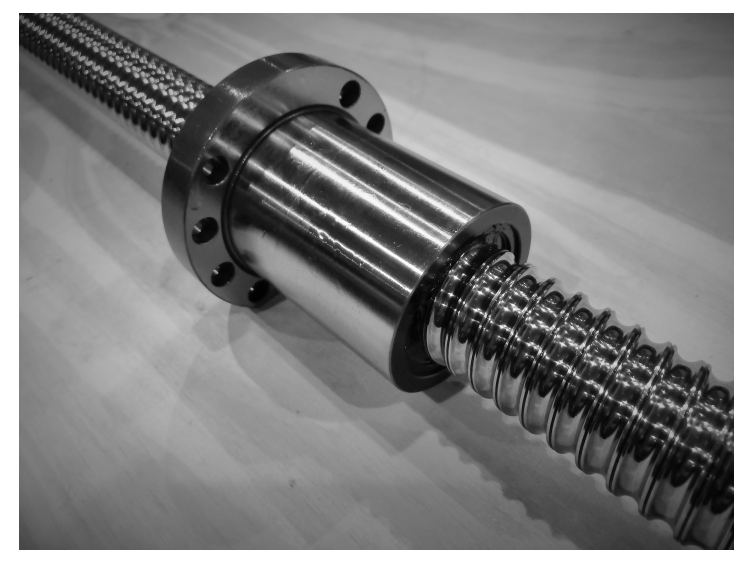

Fig. 1. Investigated machine tool ball screw and nut.

motors. The most frequently used systems today are the latter in combination with ball screws which translate the motor's rotation into a translational movement of the table. The widespread application of ball screws is due to their high efficiency, low wear and high service life [4]. The investigated ball screw is shown in figure 1 .

\section{A. Ball screw preload and degradation}

One decisive feature of a ball screw is the adjustment of preload on the ball screw nut, which is a critical property for axial positioning accuracy and the prevention of backlash. Preload can be adjusted by either adjusting a spacer in the nut, creating offset in the lead of the nut or injecting oversize balls [4]. However, over time and usage in manufacturing processes, a machine tool's ball screw degrades. In particular, abrasive wear, pitting and cracks can cause the loss of preload of the ball screw's nut and lead to lower stiffness. This lowers position accuracy, which eventually causes scraped workpieces [5], [6], [7], [8].

The use case for ball screw condition monitoring considered in this paper is based on a user decision for a certain ball screw tolerance class, which is suitable for the required manufacturing tolerance and which is determined by the adjusted ball screw preload. It is further assumed, that the user only has access to ball screws of different tolerance classes offered by a manufacturer, hence cannot continuously adjust a ball screw's 


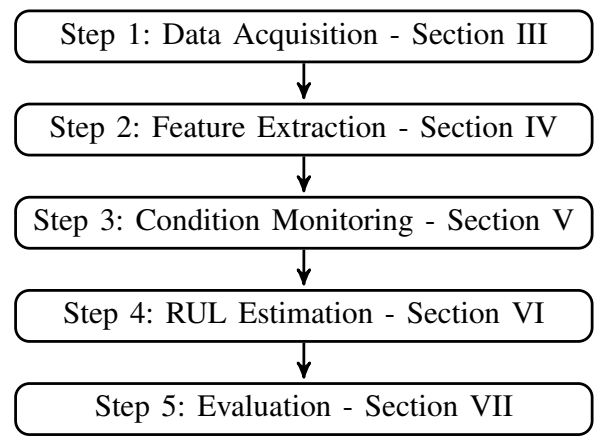

Fig. 2. Prognostic process steps based on [9].

preload. A probabilistic classification approach for estimating a ball screw's condition and RUL based on the degradation of preload will be introduced, which gives the user a basis of decision-making when to replace the components.

\section{B. Structure of the paper}

This paper's structure follows a generic prognostics process presented in [9]. It consists of data acquisition, feature extraction, condition monitoring, the estimation of RUL and the evaluation of results (see figure 2). Furthermore, this paper's focus lies on steps three and four. After giving a short overview on the state of the art in condition monitoring and RUL estimation in section II, the experimental setup and data acquisition will be shown in section III. Feature extraction will be discussed in more detail in section IV. Based on the extracted features a probabilistic approach to condition monitoring and RUL estimation will be presented in section $\mathrm{V}$ and VI respectively. The results will be discussed in section VII.

\section{CONDITION MONITORING AND RUL ESTIMATION}

In literature, condition monitoring and the estimation of RUL has been studied for a broad range of components. In general, the methods applied can be categorized into physics-based, statistics-based, artificial intelligence (AI) and hybrid approaches. The majority of contributions is applying statistics-based and AI approaches, whereas physics-based and hybrid approaches are less common in the literature [10]. In the following, statistical and AI approaches will be jointly described and treated as data-driven approaches.

Physics-based approaches rely on mathematical descriptions of the underlying physical degradation process. This can be challenging since a complete formulation of the system's behavior requires a broad and fundamental understanding, which may not be present. A selection of common physicsbased approaches are reviewed in [11] and [12]. Examples for physics-based approaches are the application of Taylor's law [13], finite element methods [14] and crack growth models such as Paris' law [15]. Often, however, the physical models' system state is identified and updated with the help of statistical methods such as Kalman filters and particle filters [16], [17]. Nonetheless, those approaches are referred to as physics-based approaches [18].
Data-driven approaches, in contrast, do not rely on a physical description of the underlying degradation process, but apply historical data to establish a relationship between observed damage and extracted damage-sensible features. An extended review on data-driven approaches can be found in [9], [19] and [12]. Exemplary models that were applied in the literature are proportional hazard models [20], Hidden Markov Models [21] and artificial neural networks [22].

Finally, hybrid approaches try to leverage the advantage of the former two by combining them in a sensible way. In [18] a detailed and structured review, as well as an exemplary application to the estimation of battery RUL with a hybrid approach, is given. They show that it can be beneficial to follow a hybrid approach compared to a pure physics-based approach.

When it comes to the estimation of RUL of ball screws, various of the above three mentioned approaches can be found in the literature. Although not every single approach can be categorized distinctly, an attempt to do so will be made in the following.

Physics-based approaches include [23], who simulated the degradation of ball screw preload and compared the result with experimental tests obtained from their test bed. More empirical examples include [24] who constructed a ball screw test bench equipped with a preload adjustable ball screw nut. After setting up an analytical model for the linear feed drive they identified the frequency ranges in which the system's eigenfrequencies can be expected to be found. Then they excited the system by commanding a signal with constant speed and linear acceleration on a stroke of $400 \mathrm{~mm}$. The recorded signals were investigated in the theoretically derived frequency ranges by estimating the eigenfrequencies as the peaks in the Fast Fourier Transform (FFT) of the system's response. They showed that preload degradation can be observed in the extracted eigenfrequencies from vibration data.

However, since eigenfrequencies are position dependent, [8] constructed a preload feature that is less dependent on the ball screw nut's position and can be successfully applied for condition monitoring.

Another physics-based approach was followed by [25] who built an analytical model for the ball passing frequency (BPF) in the ball screw nut. Based on that BPF they applied a VoldKalman filter to track the ball screw's preload. They concluded that their approach is an applicable and practical one with good results. However, in preparation of this paper, the utilization of BPF did not yield the same good results.

A data-driven approach is proposed by [26], who transformed the time domain vibration signal with the HilbertHuang-Transformation (HHT), which is a rather novel technique to analyse non-stationary and non-linear time series [27], and monitored the preload condition.

An other approach was followed by [28] who utilized several data-driven techniques in one condition monitoring pipeline to monitor not only a ball screw's condition but the condition of an entire machine tool in an unsupervised manner. They recorded vibration data first and estimated the 
signal's power spectral density (PSD). From this PSD they extracted abstract features by applying non-negative matrix factorization. Based on those features, they applied a Hidden Markov model, which estimates the current and likely future machine tool conditions.

Another approach, followed by [29], is the extensive search for failure-sensitive features as a first step. This was done by constructing as many features from vibration data as possible. Exemplary ones were summary statistics of the raw time signal such as minimum, maximum and mean value. But also features from vibration data were constructed. For example the wavelet or spectrum energy and the BPF. In step two, the main features were selected by applying a support vector machine (SVM), which used a combination of features and tried to classify the data correctly according to the true condition. Eventually, by adding features, the misclassification rate ceased to decrease any further. This is when an optimal set of features was found. They then used the set of optimal features and constructed a so-called health value, which is a combination of the set of optimal features and was labelled as one during the first operating hours and was labelled as zero during the last operating hours. All data points in between were labelled by linear interpolation. Therefore, for every point in time, the features can be measured and a health value can be evaluated. They then showed how RUL as a function of time can be estimated by applying a Gaussian process regression (GPR) model. Although this approach is rather holistic since it covers all process steps from figure 2, this paper follows another procedure, which is operating without a health value and linearity assumption.

We argue, that a hybrid approach, that combines the advantages of physical and statistical modelling equally, has not been presented for the estimation of a machine tool's ball screw RUL yet. This paper tries to fill the gap with a novel idea of estimating remaining useful life via probabilistic classification based on eigenfrequencies extracted from vibration data.

\section{DATA Acquisition: Experimental Setup}

All experiments were conducted on a DMG duo Block DMC 55H five-axis milling machine (see figure 3) equipped with a Heidenhain iTNC530 computer numeric control (CNC), which facilitates the excitement of the linear drives through defined sine sweeps within a certain frequency range. All measurements were conducted with piezo-electric Kistler 8762A10 acceleration sensors and processed with a National Instruments NI USB-4431 data acquisition box. The measurements were taken on a specific location shown in figure 3. This particular position was selected with the help of a FEM simulation model, that was developed by [30]. This simulation model accurately evaluates the machine's structural modes and therefore supports the selection of measurement positions that give optimal signals which capture relevant ball screw modes. All measurements were sampled with $20 \mathrm{kHz}$. The investigated components were Bosch Rexroth ball screws FEM-EC 40x16Rx6-4 with different preload levels. In this paper, results based on two different components are presented. First,

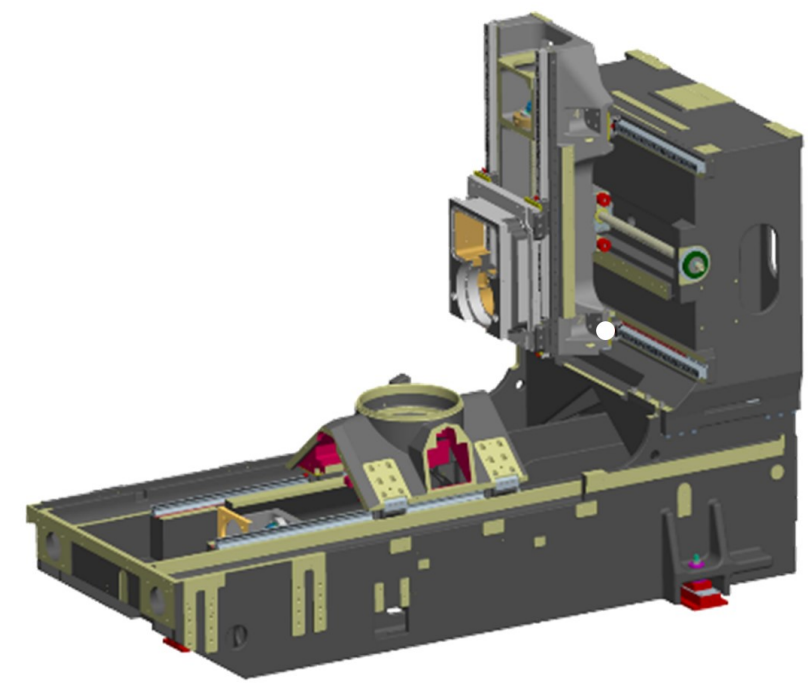

Fig. 3. Test bed. Measurement location marked as white dot.

a ball screw with $2390 \mathrm{~N}$ preload and second a ball screw with $1450 \mathrm{~N}$ preload were tested. It is assumed that the ball screw with higher preload corresponds to a healthy component which is able to fulfil the manufacturing tolerances whereas the ball screw with lower preload corresponds to a degraded component.

\section{Feature Extraction: Modal Parameters}

Assuming a linear time-invariant multi degree of freedom system with mass matrix $\mathbf{M}$, damping matrix $\mathbf{D}$, stiffness matrix $\mathbf{K}$, excitation $\mathbf{f}$ and $\ddot{\mathbf{x}}, \dot{\mathbf{x}}, \mathbf{x}$ being acceleration, velocity and position respectively [31],

$$
\mathbf{M} \ddot{\mathbf{x}}+\mathbf{D} \dot{\mathbf{x}}+\mathbf{K x}=\mathbf{f} .
$$

the frequency response function (FRF) can be described as a sum of $n$ single degree of freedom systems' FRFs:

$$
\begin{aligned}
H(\omega) & =\sum_{i=1}^{n} H_{i}(\omega)=\sum_{i=1}^{n} \frac{X_{i}(\omega)}{F(\omega)} \\
& =\sum_{i=1}^{n} \frac{1 / m_{i}}{\omega_{e, i}^{2}-\omega^{2}+j 2 D_{i} \omega_{e, i} \omega},
\end{aligned}
$$

with mass $m_{i}$, eigenfrequency $\omega_{e, i}$ and damping $D_{i}$. The studied system was excited by a CM test cycle consisting of a sine sweep on the motor current via the Heidenhain CNC. The system's response $X(\omega)$ was measured at a certain position (see figure 3). Assuming constant input $F(\omega)$ over the relevant frequency range from $1-500 \mathrm{~Hz}$, the outputs were transferred into the frequency domain via FFT and treated as the true FRF. From the system's response $X(\omega)$, eigenfrequencies were manually extracted by detecting the relevant peaks. Since eigenfrequencies are proportional to the system's stiffness [24] they were considered good features for monitoring loss in preload. Potential non-linear behaviour was controlled by always exciting the system with a standardized $\mathrm{CM}$ test cycle. Figure 4 shows two exemplary measurements. 


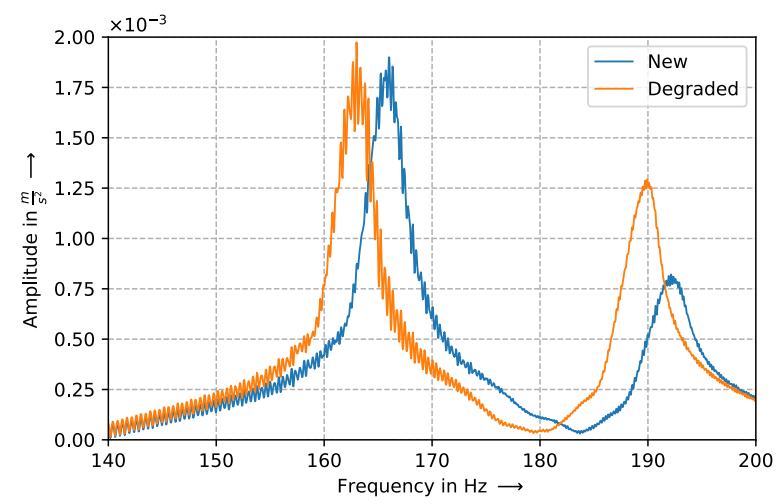

Fig. 4. Exemplary measurements of new and degraded ball screws. The loss in preload can be detected by the loss in stiffness and therefore lower eigenfrequencies.

\section{Condition Monitoring: Gaussian Process Classification}

For monitoring the condition of the ball screw, a binary classification model was applied. Due to the fact that the user cannot buy a ball screw with any preload on a continuous scale but has to decide for a ball screw in a certain tolerance class with a certain pre-adjusted preload. Hence, the condition to be monitored (preload) is a discrete value. Therefore, we decided to apply classification instead of regression.

A Gaussian process classification (GPC) was applied for several reasons. Firstly, a limited amount of observations is present. More precisely, only $n=5$ observations from $c=2$ classes were available. It is, therefore, necessary to work with methods, that can cope with little data. In GPC, this is possible through the induction of prior knowledge into the model. Secondly, GPC not only produces a point estimate of class membership for a certain ball screw but an entire probability distribution to be in a certain class. This is beneficial because it is possible to assess uncertainty around the estimation of class membership. In the case of high uncertainties, the user might ask for more information instead of making a decision. A third advantage of GPC, which is also based on assessing the probability of class membership, will become useful in section VI when the target is to estimate RUL.

In the following, Gaussian processes (GPs) and Gaussian process regression (GPR) will shortly be reviewed in accordance with [32]. Gaussian processes (GPs) are distributions over functions. Formally, if a function $f(\mathbf{x})$ follows a Gaussian process, i.e.

$$
f(\mathbf{x}) \sim \mathcal{G P}\left(m(\mathbf{x}), k\left(\mathbf{x}, \mathbf{x}^{\prime}\right)\right),
$$

with mean function $m(\mathbf{x})$, covariance function $k\left(\mathbf{x}, \mathbf{x}^{\prime}\right)$ and expectation $E$,

$$
\begin{aligned}
m(\mathbf{x}) & =E[f(\mathbf{x})] \\
k\left(\mathbf{x}, \mathbf{x}^{\prime}\right) & =E\left[(f(\mathbf{x})-m(\mathbf{x}))\left(f\left(\mathbf{x}^{\prime}\right)-m\left(\mathbf{x}^{\prime}\right)\right)\right],
\end{aligned}
$$

then any finite evaluation of a Gaussian process is a collection of jointly multivariate normal distributed random variables.
Therefore, a Gaussian process can be seen as the generalization of the multivariate Gaussian distribution. The above mentioned prior knowledge can be incorporated through the mean and covariance functions. Often the mean function is set to zero [32]. Hence, the covariance function is the remaining ingredient which can be tweaked in order to represent prior knowledge. And indeed, different covariance functions lead to different results. They can even be recombined to form new covariance functions [33]. This property makes GPs very versatile, flexible and attractive to use for modelling.

In the GPR case noisey observations $y_{i}=f\left(\mathbf{x}_{\mathbf{i}}\right)+\epsilon_{i}$ are assumed, with $\epsilon_{i}$ being Gaussian noise. One main advantage of GPR is the analytical tractability of the calculation of the posterior densities of the latent function $f=f(\mathbf{x})$, sometimes also called predictive distribution. This is due to the Gaussian assumptions on $f$ and the noise $\epsilon_{i}$. At a point of interest $f^{\star}$ at a given location $\mathbf{x}^{\star}$, given historical observations $\mathbf{y}$ at historical input locations $\mathbf{x}$, the predictive distribution is given by the conditional density $p\left(f^{\star} \mid \mathbf{x}, \mathbf{y}, \mathbf{x}^{\star}\right)=$ $\mathcal{N}\left(f^{\star} \mid E\left(f^{\star} \mid \mathbf{x}, \mathbf{y}, \mathbf{x}^{\star}\right), \operatorname{Var}\left(f^{\star} \mid \mathbf{x}, \mathbf{y}, \mathbf{x}^{\star}\right)\right)$ with

$$
\begin{aligned}
E\left(f^{\star} \mid \mathbf{x}, \mathbf{y}, \mathbf{x}^{\star}\right) & =k\left(\mathbf{x}^{\star}, \mathbf{x}\right)\left[k(\mathbf{x}, \mathbf{x})+\sigma_{n}^{2} I\right]^{-1} \mathbf{y}, \\
\operatorname{Var}\left(f^{\star} \mid \mathbf{x}, \mathbf{y}, \mathbf{x}^{\star}\right) & =k\left(\mathbf{x}^{\star}, \mathbf{x}^{\star}\right) \\
& -k\left(\mathbf{x}^{\star}, \mathbf{x}\right)\left[k(\mathbf{x}, \mathbf{x})+\sigma_{n}^{2} I\right]^{-1} k\left(\mathbf{x}, \mathbf{x}^{\star}\right),
\end{aligned}
$$

where $\sigma_{n}^{2}$ is the measurement noise of each observation and $I$ is the identity matrix. An exemplary covariance function is the squared exponential (SE) covariance function [32]:

$$
k\left(\mathbf{x}, \mathbf{x}^{\prime}\right)_{\mathrm{SE}}=\sigma_{\mathrm{SE}}^{2} \exp \left(-\frac{\left\|\mathbf{x}-\mathbf{x}^{\prime}\right\|^{2}}{2 l^{2}}\right),
$$

with so-called hyper-parameters $\sigma_{\mathrm{SE}}$ and $l$. In the setting of classification, it is assumed, that the latent data generating process is a Gaussian process. It is further assumed, that the latent Gaussian process is squashed through a probit function, which ensures the function values to be between zero and one, which is the probability of belonging to a certain class. However, in GPC the predictive distribution is not analytically tractable any more due to the non-Gaussian likelihood caused by the probit function. Therefore, one has to apply approximations like the Laplace approximation, expectation propagation (EP) or Markov Chain Monte Carlo (MCMC) sampling [34], [32]. In this paper inference was conducted using the EP algorithm since it yields results close to MCMC methods, which provide exact results in the limit of infinite runs and therefore can be seen as a benchmark [34]. The idea behind EP is to approximate the true non-Gaussian predictive distribution $p(\cdot)$ with a Gaussian distribution $q(\cdot) \sim \mathcal{N}(\tilde{\boldsymbol{\mu}}, \tilde{\Sigma})$. Assuming appropriate parameters for $q(\cdot)$ can be found, the solution for the approximated predictive distribution of the latent function derived with the EP algorithm is $q\left(f^{\star} \mid \mathbf{x}, \mathbf{y}, \mathbf{x}^{\star}\right)=$ 

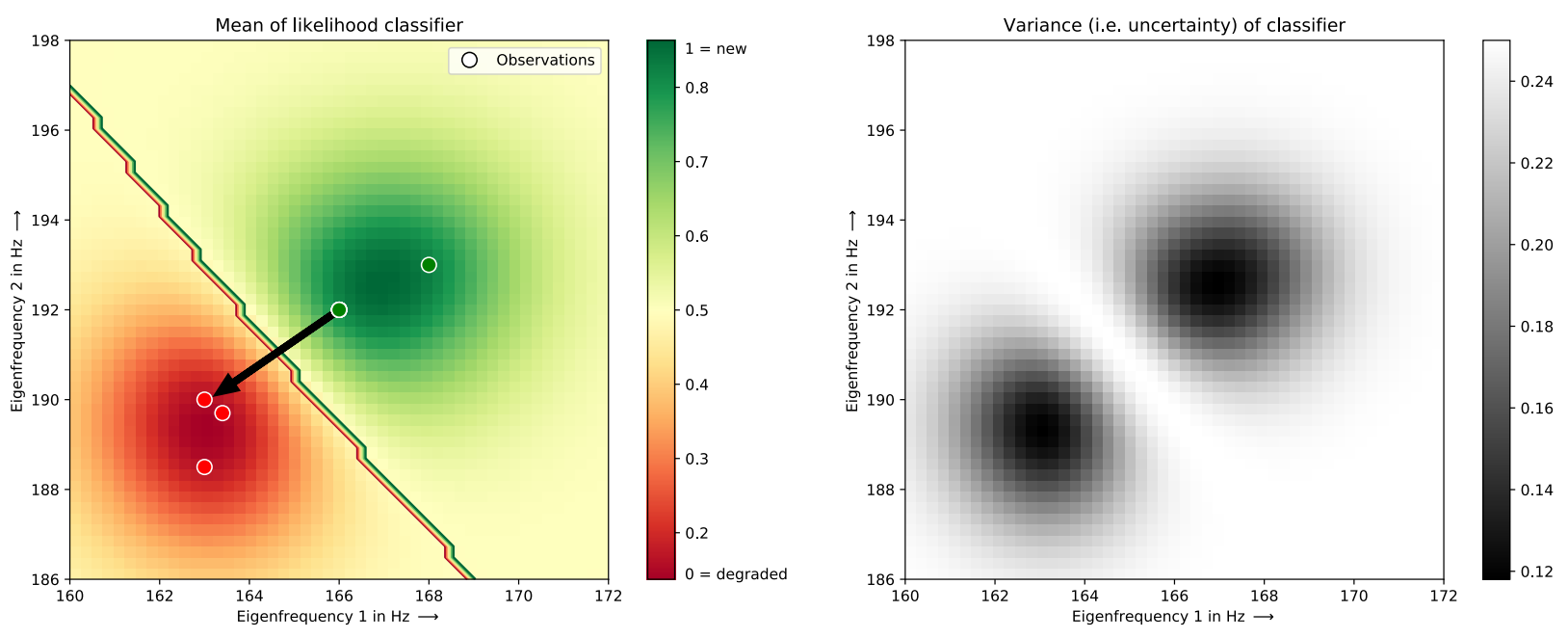

Fig. 5. GPC with RBF covariance function. Left is the mean class probability and right the variance (i.e. uncertainty) around the mean estimate.

$$
\begin{aligned}
& \mathcal{N}\left(f^{\star} \mid E_{q}\left(f^{\star} \mid \mathbf{x}, \mathbf{y}, \mathbf{x}^{\star}\right), \operatorname{Var}_{q}\left(f^{\star} \mid \mathbf{x}, \mathbf{y}, \mathbf{x}^{\star}\right)\right)[35],[34] \text { with } \\
& E_{q}\left(f^{\star} \mid \mathbf{x}, \mathbf{y}, \mathbf{x}^{\star}\right)=k\left(\mathbf{x}, \mathbf{x}^{\star}\right)^{\top}\left(k(\mathbf{x}, \mathbf{x})+\tilde{\Sigma}^{-1}\right)^{-1} \tilde{\Sigma}^{-1} \tilde{\boldsymbol{\mu}}, \\
& \operatorname{Var}_{q}\left(f^{\star} \mid \mathbf{x}, \mathbf{y}, \mathbf{x}^{\star}\right)=k\left(\mathbf{x}^{\star}, \mathbf{x}^{\star}\right) \\
&-k\left(\mathbf{x}, \mathbf{x}^{\star}\right)^{\top}(k(\mathbf{x}, \mathbf{x})+\tilde{\Sigma})^{-1} k\left(\mathbf{x}, \mathbf{x}^{\star}\right) .
\end{aligned}
$$

The approximate predictive probability for a new observation $y^{\star}$ belonging to class one can then be found with

$$
\begin{aligned}
& q\left(y^{\star}=1 \mid \mathbf{x}, \mathbf{y}, \mathbf{x}^{\star}\right)=\int \Phi\left(f^{\star}\right) q\left(f^{\star} \mid \mathbf{x}, \mathbf{y}, \mathbf{x}^{\star}\right) d f^{\star} \\
= & \Phi\left(\frac{k\left(\mathbf{x}, \mathbf{x}^{\star}\right)^{\top}(k(\mathbf{x}, \mathbf{x})+\tilde{\Sigma})^{-1} \tilde{\boldsymbol{\mu}}}{\sqrt{1+k\left(\mathbf{x}^{\star}, \mathbf{x}^{\star}\right)-k\left(\mathbf{x}, \mathbf{x}^{\star}\right)^{\top}(k(\mathbf{x}, \mathbf{x})+\tilde{\Sigma})^{-1} k\left(\mathbf{x}, \mathbf{x}^{\star}\right)}}\right),
\end{aligned}
$$

where $\Phi$ is the probit function and $\tilde{\mu}$ and $\tilde{\Sigma}$ are the mean vector and covariance matrix of the approximate posterior $q(\cdot)$.

In this paper an RBF covariance function, as in equation 6, was used for the GPC model and the hyper-parameters were estimated applying the type II maximum likelihood (ML-II) approach. The resulting probabilistic classification model is shown in figure 5 . The model gives a hard decision boundary, which divides new and degraded ball screws into two classes. The degraded ball screws are shown in the lower left corner and the new ball screws are shown in the upper right corner. The model is quite confident in classifying a given ball screw based on its eigenfrequencies in areas where historical observations are available. In areas where this is not the case, the model makes a guess but is also less certain. This is intuitive, sensible and supports the user in decision-making. Furthermore, the uncertainties around the estimates are quantified, which supports the user additionally. The uncertainty is high where historical observations are absent and small where observations were available. However, the uncertainty is not only dependent on the availability of historical data but also dependent on the degree of the model assumptions matching the observed data. Loosely spoken, the model is implicitly giving feedback on its own confidence of representing the observed data, which again is supporting the decision making process.

\section{RUL Estimation: Gaussian Process Regression}

Finally, the target is to calculate RUL for the critical component ball screw. However, since the user can only monitor class membership with respect to those tolerance classes and preload adjustments a ball screw manufacturer offers, calculating RUL is a challenging task. In order to extrapolate degradation and estimate RUL until a critical threshold is met, continuous values are necessary. Here, the GPC model from the previous section becomes handy, since in that model not only class membership as a discrete variable is estimated but also the probability of belonging to a class. This probability is a continuous value between zero and one and can, therefore, be extrapolated with common regression methods. In the following, we apply a GPR model to estimate RUL. It is important to note, that the data used is simulated from the GPC in figure 5. It was assumed that a new ball screw degrades over time along the black arrow and eventually can be considered worn out. If this is true, then measurements over time will look like the observations in figure 6. At time zero, the ball screw can be considered new, equipped with high preload, and thus higher eigenfrequencies. Consequently, the GPC in figure 5 assesses a high probability for the ball screw to actually be a new ball screw. Over time and usage, the ball screw experiences degradation. At random points in time CM test cycles are conducted (as described in section III), noisy measurements collected, the eigenfrequencies analysed, degradation with the help of the GPC assessed and the likelihood of class membership entered into figure 6 . As expected a monotonic degradation can be observed with increasing operating time.

The degradation pathway is modelled and extrapolated with 
a GPR model as described in section V. Now, however, with subscript time $t$ and one-dimensional inputs $x_{t}$ only.

It is assumed that degradation over time is observed in form of the mean probability of class membership $y_{t}$, which in return is assumed to be a noisy realization that follows a latent function $f\left(x_{t}\right) \sim \mathcal{G P}\left(0, k\left(x, x^{\prime}\right)_{\mathrm{RUL}}\right)$. Formally:

$$
y_{t}=f\left(x_{t}\right)+\epsilon_{t}, \quad \epsilon_{t} \sim \mathcal{N}\left(0, \sigma_{\epsilon}^{2}\right) .
$$

Since a ball screw's loss in preload is assumed to be monotonic and non-stationary, it was decided to use an additive, nonstationary covariance function consisting of three components [36]. Firstly, a non-stationary polynomial component (Poly) that can capture the degradation process' negative growth trend. Secondly, a smoothing component (SE) that softens the strict, parametric polynomial influence, and thirdly, a noise component (WN) that can capture the measurement noise coming from the vibration measurements. Hence, the covariance function is:

$$
k\left(x, x^{\prime}\right)_{\mathrm{RUL}}=k\left(x, x^{\prime}\right)_{\mathrm{SE}}+k\left(x, x^{\prime}\right)_{\mathrm{WN}}+k\left(x, x^{\prime}\right)_{\mathrm{Poly}},
$$

with

$$
\begin{aligned}
& k\left(x, x^{\prime}\right)_{\mathrm{SE}}=\sigma_{\mathrm{SE}}^{2} \exp \left(-\frac{\left(x-x^{\prime}\right)^{2}}{2 l^{2}}\right), \\
& k\left(x, x^{\prime}\right)_{\mathrm{WN}}=\sigma_{\mathrm{WN}}^{2} \mathrm{I}, \quad \mathrm{I}=\left\{\begin{array}{ll}
1 & \text { if } x=x^{\prime} \\
0 & \text { otherwise }
\end{array},\right. \\
& k\left(x, x^{\prime}\right)_{\text {Poly }}=\left[(x-c)\left(x^{\prime}-c\right)\right]^{2} .
\end{aligned}
$$

For inferring the hyper-parameters $\sigma_{\mathrm{SE}}, \sigma_{\mathrm{WN}}, l$ and $c$ from data the ML-II method was applied.

It can be seen from figure 6 that the monotonic degradation process of the ball screw is captured by the GPR model. Not only the in-sample fit is well, but also the extrapolated behaviour is close to the true behaviour for a long time ahead. In fact, the RUL in this example, which is the time from the last simulated measurement until the threshold of $E\left[q\left(y_{t}=1\right)\right]=0.5$ is met, is almost perfectly estimated. Although, the uncertainty around the estimate grows with time. This is sensible, however, since the further away from observations one moves, the more uncertain one should be about the prediction.

\section{CONCLUSION AND OUTLOOK}

This paper presented a new idea to estimate RUL of machine tool ball screws via probabilistic classification. After giving an introduction and a brief literature review, a new hybrid approach was presented using a combination of physics-based and data-driven methods.

The presented approach is based on the ball screws eigenfrequencies extracted from vibration data. Based on the eigenfrequencies, a ball screw can be classified in tolerance classes provided by a ball screw manufacturer. This paper showed an example with real data that classified a ball screw into two tolerance classes, one of which was considered in working order while the other was considered degraded. The classification

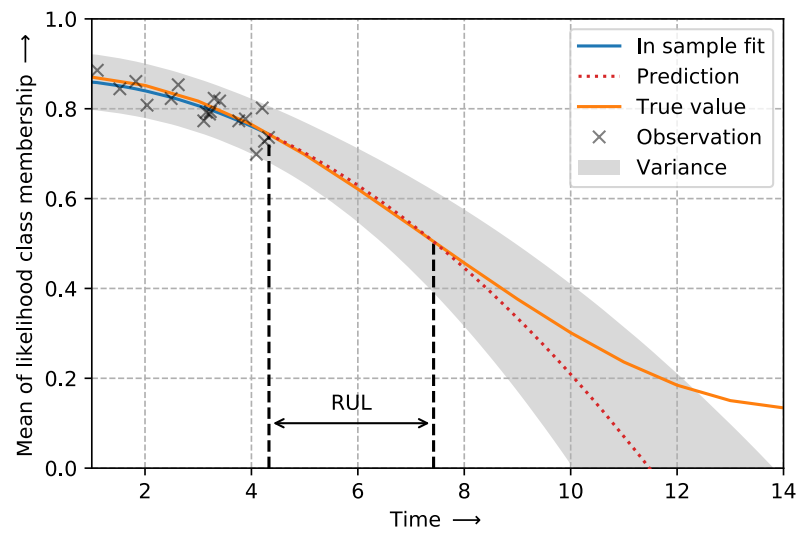

Fig. 6. Estimated RUL. The threshold was set to the point, where the probability of being a new ball screw is equal to $50 \%$.

was performed with a GPC model, which is a probabilistic method, that gives the probability of class membership. This is beneficial for two reasons. Firstly, uncertainties in classification are quantified and can be considered for decision making and secondly, since probabilities are, in contrast to classes, continuous variables, extrapolation becomes possible. In the end this extrapolation lead to the estimation of RUL, i.e. the time a threshold is predicted to be met.

However, the presented approach still needs more validation to be considered entirely feasible. Firstly, more measurements need to be conducted that can validate the current approach and take into account more components such as linear guides and more tolerance classes than two. Secondly, a more autonomous way of extracting eigenfrequencies must be implemented. Currently, eigenfrequencies are identified manually. Thirdly, more probabilistic classification models need to be considered and compared with respect to the ability of correctly modelling the degradation. Fourthly, long-term trials need to be conducted to assess the accuracy of the RUL estimation. Fifthly, RUL should be treated as a function of time and load in order to be useful for maintenance integrated production scheduling [37]. Currently RUL is only treated as a function of time.

\section{ACKNOWLEDGMENT}

This project has received funding from the European Union's Horizon 2020 research and innovation programme under grant agreement No. 768575 .

\section{REFERENCES}

[1] Y. K. Son, "A cost estimation model for advanced manufacturing systems," International Journal of Production Research, vol. 29, no. 3, pp. 441-452, Mar. 1991.

[2] A. Widodo and B.-S. Yang, "Support vector machine in machine condition monitoring and fault diagnosis," Mechanical Systems and Signal Processing, vol. 21, no. 6, pp. 2560-2574, Aug. 2007.

[3] B. Schmidt, "Cloud-enhanced predictive maintenance," Int J Adv Manuf Technol, p. 9, 2016.

[4] Y. Altintas, A. Verl, C. Brecher, L. Uriarte, and G. Pritschow, "Machine tool feed drives," CIRP Annals, vol. 60, no. 2, pp. 779-796, 2011. 
[5] G. W. Vogl, M. A. Donmez, and A. Archenti, "Diagnostics for geometric performance of machine tool linear axes," p. 16, 2017.

[6] Y. Zhou, X. Mei, Y. Zhang, G. Jiang, and N. Sun, "Current-based Feed Axis Condition Monitoring and Fault Diagnosis," p. 5, 2009.

[7] E. Uhlmann, C. Geisert, and E. Hohwieler, "Monitoring of slowly progressing deterioration of computer numerical control machine axes," Part B, vol. 222, p. 7, 2008.

[8] T. L. Nguyen, S.-K. Ro, C. K. Song, and J.-K. Park, "Study on Preload Monitoring of Ball Screw Feed Drive System Using Natural Frequency Detection," Journal of the Korean Society for Precision Engineering, vol. 35, no. 2, pp. 135-143, Feb. 2018.

[9] R. Li, W. J. Verhagen, and R. Curran, "A comparative study of Data-driven Prognostic Approaches: Stochastic and Statistical Models," in 2018 IEEE International Conference on Prognostics and Health Management (ICPHM). Seattle, WA, USA: IEEE, Jun. 2018, pp. 1-8.

[10] Y. Lei, N. Li, L. Guo, N. Li, T. Yan, and J. Lin, "Machinery health prognostics: A systematic review from data acquisition to RUL prediction," Mechanical Systems and Signal Processing, vol. 104, pp. 799-834, May 2018.

[11] A. Heng, S. Zhang, A. C. Tan, and J. Mathew, "Rotating machinery prognostics: State of the art, challenges and opportunities," Mechanical Systems and Signal Processing, vol. 23, no. 3, pp. 724-739, Apr. 2009.

[12] R. Gao, L. Wang, R. Teti, D. Dornfeld, S. Kumara, M. Mori, and M. Helu, "Cloud-enabled prognosis for manufacturing," CIRP Annals, vol. 64, no. 2, pp. 749-772, 2015.

[13] B. Mills and A. H. Redford, Machinability of Engineering Materials. Dordrecht: Springer Netherlands, 1983.

[14] C. J. Li and H. Lee, "Gear fatigue crack prognosis using embedded model, gear dynamic model and fracture mechanics," Mechanical Systems and Signal Processing, vol. 19, no. 4, pp. 836-846, Jul. 2005.

[15] N. Pugno, M. Ciavarella, P. Cornetti, and A. Carpinteri, "A generalized Paris' law for fatigue crack growth," Journal of the Mechanics and Physics of Solids, vol. 54, no. 7, pp. 1333-1349, Jul. 2006.

[16] D. C. Swanson, J. Michael Spencer, and S. H. Arzoumanian, "Prognostics Modelling of Crack Growth in a Tensioned Steel Band," Mechanical Systems and Signal Processing, vol. 14, no. 5, pp. 789-803, Sep. 2000.

[17] M. Daigle and K. Goebel, "Improving Computational Efficiency of Prediction in Model-based Prognostics Using the Unscented Transform," p. 12, 2010.

[18] L. Liao and F. Köttig, "Review of Hybrid Prognostics Approaches for Remaining Useful Life Prediction of Engineered Systems, and an Application to Battery Life Prediction," IEEE TRANSACTIONS ON RELIABILITY, vol. 63, no. 1, p. 17, 2014.

[19] X.-S. Si, W. Wang, C.-H. Hu, and D.-H. Zhou, "Remaining useful life estimation - A review on the statistical data driven approaches," European Journal of Operational Research, vol. 213, no. 1, pp. 1-14, Aug. 2011.

[20] V. T. Tran, H. Thom Pham, B.-S. Yang, and T. Tien Nguyen, "Machine performance degradation assessment and remaining useful life prediction using proportional hazard model and support vector machine," Mechanical Systems and Signal Processing, vol. 32, pp. 320-330, Oct. 2012.

[21] C. Bunks, D. Mccarthy, and T. Al-Ani, "Condition-based Maintenance of Machines Using Hidden Markov Models," Mechanical Systems and Signal Processing, vol. 14, no. 4, pp. 597-612, Jul. 2000.

[22] Z. Tian, "An artificial neural network method for remaining useful life prediction of equipment subject to condition monitoring," Journal of Intelligent Manufacturing, vol. 23, no. 2, pp. 227-237, Apr. 2012.

[23] D. Maier and U. Heisel, "A comparision of model and signal based condition monitoring and mode separation for predictive maintenance of feed drives," Journal of Machine Engineering, vol. Vol. 11, No. 4, pp. 138-151, 2011.

[24] G.-H. Feng and Y.-L. Pan, "Investigation of ball screw preload variation based on dynamic modeling of a preload adjustable feed-drive system and spectrum analysis of ball-nuts sensed vibration signals," International Journal of Machine Tools and Manufacture, vol. 52, no. 1, pp. 85-96, Jan. 2012.

[25] P. Tsai, C. Cheng, and Y. Hwang, "Ball screw preload loss detection using ball pass frequency," Mechanical Systems and Signal Processing, vol. 48, no. 1-2, pp. 77-91, Oct. 2014.

[26] Y.-C. Huang and Y.-C. Shin, "Method of Intelligent Fault Diagnosis of Preload Loss for Single Nut Ball Screws through the Sensed Vibration Signals," vol. 6, no. 5, p. 8, 2012.

[27] N. E. Huang, "Introduction to the Hilbert Huang Transform and its related mathematical problems," p. 26.
[28] D. Kißkalt, H. Fleischmann, S. Kreitlein, M. Knott, and J. Franke, "A novel approach for data-driven process and condition monitoring systems on the example of mill-turn centers," Production Engineering, vol. 12, no. 3-4, pp. 525-533, Jun. 2018.

[29] P. Li, X. Jia, J. Feng, H. Davari, G. Qiao, Y. Hwang, and J. Lee, "Prognosability study of ball screw degradation using systematic methodology," Mechanical Systems and Signal Processing, vol. 109, pp. 45-57, Sep. 2018.

[30] C. Rebelein, T. Semm, and M. F. Zäh, "Simulation of Damping Effects in Machine Tool Structures," ZWF Zeitschrift für wirtschaftlichen Fabrikbetrieb, vol. 112, no. 11, pp. 736-740, Nov. 2017.

[31] Y. Altintas, Manufacturing automation: metal cutting mechanics, machine tool vibrations, and CNC design, 2nd ed. Cambridge ; New York: Cambridge University Press, 2012, oCLC: ocn751750911.

[32] C. Rasmussen and C. Williams, Gaussian Processes for Machine Learning, ser. Adaptive Computation and Machine Learning. Cambridge, MA, USA: MIT Press, 2006.

[33] C. M. Bishop, Pattern recognition and machine learning, ser. Information science and statistics. New York: Springer, 2006.

[34] M. Kuss and C. E. Rasmussen, "Assessing Approximate Inference for Binary Gaussian Process Classification,” p. 26, 2005.

[35] T. E. Minka, "Expectation Propagation for Approximate Bayesian Inference," p. 8, 2001.

[36] D. Duvenaud, H. Nickisch, and C. E. Rasmussen, "Additive Gaussian Processes," arXiv:1112.4394 [cs, stat], Dec. 2011, arXiv: 1112.4394.

[37] S. Zhai and G. Reinhart, "Predictive Maintenance as an Enabler for Maintenance-integrated Production Planning," ZWF Zeitschrift für wirtschaftlichen Fabrikbetrieb, vol. 113, no. 5, pp. 298-301, May 2018. 